\title{
Altered Functional Connectivity Following an Inflammatory White Matter Injury in the Newborn Rat: A High Spatial and Temporal Resolution Intrinsic Optical Imaging Study
}

\begin{abstract}
Edgar Guevara ${ }^{1 *}$, Wyston C. Pierre², Camille Tessier², Luis Akakpo², Irène Londono², Frédéric Lesage ${ }^{3,4}$ and Gregory A. Lodygensky 2,3, 5, 6

${ }^{1}$ Terahertz Science and Technology National Lab, CONACYT-Universidad Autónoma de San Luis Potosí, Coordinación para la Innovación y Aplicación de la Ciencia y la Tecnología, San Luis Potosí, Mexico, ${ }^{2}$ Sainte-Justine Hospital and Research Center, Department of Pediatrics, Université de Montréal, Montreal, QC, Canada, ${ }^{3}$ Montreal Heart Institute, Research Center, Montreal, QC, Canada, ${ }^{4}$ Department of Electrical Engineering, École Polytechnique de Montréal, Montreal, QC, Canada, ${ }^{5}$ Department of Pharmacology, Université de Montréal, Montreal, QC, Canada, ${ }^{6}$ Department of Neuroscience, Université de Montréal, Montreal, QC, Canada
\end{abstract}

OPEN ACCESS

Edited by:

Xi-Nian Zuo,

Institute of Psychology (CAS), China

Reviewed by:

Xin Di,

New Jersey Institute of Technology,

United States

Rui Li,

Institute of Psychology (CAS), China

${ }^{*}$ Correspondence:

Edgar Guevara

eguevara@conacyt.mx

Specialty section:

This article was submitted to Brain Imaging Methods,

a section of the journal

Frontiers in Neuroscience

Received: 20 January 2017

Accepted: 08 June 2017

Published: 04 July 2017

Citation:

Guevara E, Pierre WC, Tessier C, Akakpo L, Londono I, Lesage F and

Lodygensky GA (2017) Altered Functional Connectivity Following an Inflammatory White Matter Injury in the

Newborn Rat: A High Spatial and Temporal Resolution Intrinsic Optical

Imaging Study.

Front. Neurosci. 11:358.

doi: 10.3389/fnins.2017.00358
Very preterm newborns have an increased risk of developing an inflammatory cerebral white matter injury that may lead to severe neuro-cognitive impairment. In this study we performed functional connectivity (fc) analysis using resting-state optical imaging of intrinsic signals (rs-OIS) to assess the impact of inflammation on resting-state networks (RSN) in a pre-clinical model of perinatal inflammatory brain injury. Lipopolysaccharide (LPS) or saline injections were administered in postnatal day (P3) rat pups and optical imaging of intrinsic signals were obtained 3 weeks later. (rs-OIS) fc seed-based analysis including spatial extent were performed. A support vector machine (SVM) was then used to classify rat pups in two categories using fc measures and an artificial neural network (ANN) was implemented to predict lesion size from those same fc measures. A significant decrease in the spatial extent of fc statistical maps was observed in the injured group, across contrasts and seeds ( ${ }^{*} p=0.0452$ for $\mathrm{HbO}_{2}$ and ${ }^{* *} p=0.0036$ for $\mathrm{HbR}$ ). Both machine learning techniques were applied successfully, yielding 92\% accuracy in group classification and a significant correlation $r=0.9431$ in fractional lesion volume prediction $(* * p=0.0020)$. Our results suggest that fc is altered in the injured newborn brain, showing the long-standing effect of inflammation.

Keywords: white matter injury, inflammation, prematurity, resting state functional connectivity, optical imaging of intrinsic signals, support vector machines, artificial neural networks

\section{INTRODUCTION}

Functional connectivity, defined as the coordination of activity across anatomically distinct regions of the brain (Aertsen et al., 1989; Friston et al., 1993), is based on assessment of neuronal activity, either directly, through electrophysiological signals, or indirectly, via hemodynamics or metabolites fluctuations.

Functional MRI (fMRI), arguably the most commonly used technique to assess brain activation, relies on the measurement of blood-oxygen-level-dependant (BOLD) signals. It reflects changes 
of blood volume and deoxyhemoglobin (HbR) concentrations (Buxton, 2013). fMRI was first described by Ogawa in 1990 (Ogawa and Lee, 1990; Ogawa et al., 1990) and has since revolutionized human neuroscience by allowing the identification of brain areas responsible for diverse cognitive task or processing various stimuli (Belliveau et al., 1991; Raichle, 2009). Resting-state fMRI (rs-fMRI) introduced a few years later (Biswal et al., 1995) corresponds to the measurement of low frequency fluctuations $(0.009<f<0.1 \mathrm{~Hz})$ in the BOLD signal in the absence of a task or stimulus. It has also greatly contributed to the neuroscience field by revealing the existence of multiple spatially distributed large-scale networks in the brain, the resting-state networks (RSN). Lewis and al. have shown that a possible function of RSN could be related to the consolidation of previous experience (Lewis et al., 2009). According to another hypothesis, the networks functioning during active processing are maintained during rest (and therefore become the RSN) in order to allow rapid activation when needed for active processing (Smith et al., 2009). However, the true function of the RSN remains unknown.

The application of fMRI or rs-fMRI in animal models has been scarce and mostly limited to rats and monkeys, because smaller models such as mice or neonatal rats require a very high intensity magnetic field to obtain sufficient signal-tonoise ratio and spatial resolution to assess their small brains (Benveniste and Blackband, 2002; Jonckers et al., 2011). Given the tremendous number of already well-characterized models that can't be readily studied because of this limitation, interest for the other functional neuroimaging techniques, alone or in combination with fMRI, has increased over the past years (Cang et al., 2005; He and Liu, 2008; Ye et al., 2016). Optical imaging of intrinsic signals (OIS) was shown to be a potent alternative to fc-MRI, also by measuring changes in blood oxygenation (Grinvald et al., 1986; Frostig et al., 1990; Ts'o et al., 1990). In this technique, two wavelengths based on the absorption spectra of oxyhemoglobin $\left(\mathrm{HbO}_{2}\right)$ and deoxyhemoglobin $(\mathrm{HbR})$, are shone upon exposed nervous tissue and fluctuations in reflected light intensity are recorded. Those fluctuations represent changes in blood oxygenation/volume and, therefore, can be used to infer changes in neuronal activity. Advantages of OIS over (rs-)fMRI include a much higher spatial resolution (in the order of tens of micrometers for OIS vs. 200-400 $\mu \mathrm{m}$ for fMRI) and temporal resolution ( $30 \mathrm{~Hz}$ for OIS vs. $0.5 \mathrm{~s}$ for high-resolution fMRI sequences; Pouratian and Toga, 2002; Jonckers et al., 2015; Pan et al., 2015), providing an interesting tool to assess functional connectivity in small animals models. Unlike fMRI, OIS is not prone to artifacts related to mechanical vibrations and spurious responses arisen from loud acoustic stimuli. Moreover, OIS also uses non-ionizing radiation and is much more costeffective than fMRI. However, one major limitation of OIS is its inability to assess subcortical structures (Hillman, 2007). The resting state data, acquired with this technique, is hence 2 dimensional. Nevertheless, most of the RSN have some, if not all, of their brain regions in the cortex (Rosazza and Minati, 2011), making OIS a suitable method to study RSNs in small models, like rodents ( $\mathrm{Li}$ et al., 2012; Guevara et al., 2013c).
The study of alterations in RSN via rs-fMRI has improved our understanding of many neurological and psychiatric diseases such as epilepsy (Wang Z. et al., 2011; Mankinen et al., 2012; Tracy and Doucet, 2015), depression (Mulders et al., 2015), autism (Farrant and Uddin, 2016; Mevel and Fransson, 2016), schizophrenia (Sheffield and Barch, 2016), and dementia (Greicius et al., 2004; Gili et al., 2011; Peraza et al., 2015). The impact of prematurity on neurocognitive development has also been, more recently, studied through fMRI and rs-fMRI. Studies have identified resting state network maturation in the growing brain with evidence of networks as early as 30 weeks of gestational age with a fast maturation leading to adult-like network at term equivalent age (Doria et al., 2010; Smyser et al., 2010; Lee et al., 2013; van den Heuvel et al., 2015; He and Parikh, 2016). The consequences of prematurity itself was revealed by showing a decreased functional connectivity of RSN in (very preterm) VPTborn patients (Smyser et al., 2010, 2013; Ye et al., 2016), best seen using correlation and covariance matrix analyses demonstrated by Smyser et al. (2016b). In other words, the topography of RSN in VPT-born children seems preserved, but quantitative parameters, such as the synchronicity between networks (White et al., 2014; Ye et al., 2016), the amplitude of BOLD signal fluctuations (Smyser et al., 2013, 2016b) or the number of voxels in a RSN (Smyser et al., 2010), appeared to be decreased with a more pronounced reduction in higher order RSN (Default mode, executive control, and frontoparietal networks; Smyser et al., 2016b). Most studies reported that primary RSN (somatosensory, motor, and visual) are almost fully formed (i.e., adult-like) before week 30 of PMA while higher order networks mature during the last trimester (Doria et al., 2010).

The exploration of RSN in VPT children is still in its very first steps. Studies have investigated connectivity in the first 4 years of life of children born preterm (Lee et al., 2013) and in adults and older children born preterm (White et al., 2014; Ye et al., 2016). In spite of the useful information obtained through cohort studies, preclinical models are crucial to test neuroprotective treatment. However, as stated earlier, the data on rodents or other small models remain scarce because of the intrinsic limitations of fMRI. In this study, we characterized using rs-OIS, resting state networks in a preclinical model of diffuse white matter injury that consists in the intra-cerebral injection of lipopolysaccharide (LPS) (Cai et al., 2003; Pang et al., 2003; Lodygensky et al., 2010, 2014; Guevara et al., 2013a). LPS is known to mimic every hallmark of inflammatory white matter injury, in the immature brain such as seen in preterm infants following necrotizing enterocolitis or born in the context of severe chorioamnionitis e.g., hippocampal volume impairment (Wang et al., 2013), ventricle dilation, apoptosis, pre-olygodendrocite cell necrosis, white matter rarefaction, hypomyelination, microglial reaction (Cai et al., 2003; Pang et al., 2003) as well as neurobehavioral deficits (Fan et al., 2005).

\section{METHODS}

\section{Animal Model}

All procedures were sanctioned by the Institutional Committee for Animal Care in Research of the CHU Sainte-Justine and 
Montreal Heart Institute Research Centers, and conducted under isoflurane anesthesia to minimize pain and distress, following the recommendations of the Canadian Council on Animal Care.

A total of 15 Sprague-Dawley pups coming from two litters (7.96 $\pm 0.45 \mathrm{~g}$ weight, Charles River, Senneville, Qc, Canada) were randomized in two different groups: LPS $(n=6)$ which were injected at postnatal day 3 (P3, equivalent to human gestational age of about 24-28 weeks (Sizonenko et al., 2003; Lodygensky et al., 2014) with a solution of lipopolysaccharide diluted in saline (1 mg/kg in $0.5 \mu \mathrm{L}$, E. Coli, serotype 055:B5, Sigma St Louis, $\mathrm{MO})$ and a sham control group $\mathrm{NaCl}(n=9)$, injected with saline alone. Injection site was the left corpus callosum at a level equivalent to P-7, c9 (Lodygensky et al., 2014). All injections were performed with an ultrasound-guided micro injector (Micro4 from World Precision Instruments) at a rate of $0.1 \mu \mathrm{L} / \mathrm{min}$. From these rat pups 2 animals were rejected, one from each group, due to a technical error during data acquisition that was only identified later on; so the final population is LPS ( $n=5 ; 1$ female, 4 males) and $\mathrm{NaCl}$ ( $n=8 ; 2$ females, 6 males).

\section{Resting-State Optical Imaging of Intrinsic Signals (rs-OIS) \\ Animal Preparation}

At postnatal day 24 or 25 (P24 or P25, equivalent to human pre-puberty (Chen et al., 2016) the rat pups were fixed onto a small animal physiological monitoring station (Small Animal Monitoring System 75-501 Harvard Apparatus, Holliston, MA) which allowed the restriction of head motion and continuous recording of respiration, heart rate, and closed-loop thermoregulation at $37 \pm 0.5^{\circ} \mathrm{C}$.

In addition to urethane $(2 \mathrm{~g} / \mathrm{kg})$, a local anesthetic (Xylocaine $0.2 \%$ ) was injected and scalp was carefully removed to expose the skull covering the cortex. Artificial cerebrospinal fluid (Bélanger et al., 2016) was poured over the skull to prevent drying and minimize specular reflections due to skull bone. Urethane was chosen over isoflurane due to concerns of its negative impact on resting state activity (Wang $\mathrm{K}$. et al., 2011). Ideally resting state activity should be performed awake but sedation was required due to the surgery. Urethane was chosen as an alternative as it has been used and recommended in fMRI studies (Huttunen et al., 2008; Paasonen et al., 2016) and in previous resting state studies (Wilson et al., 2011; Kozberg et al., 2013).

\section{Optical Imaging}

Imaging was carried out according to previous rs-OIS studies in rodents (Guevara et al., 2013b,c; Bélanger et al., 2016). Briefly, multi-spectral rs-OIS ( $\lambda=525,590,630 \mathrm{~nm}$ ) was performed under anesthesia. Seven minutes of resting state activity were recorded. Time multiplexed illumination (3.5W LED, LZ400MA00, Led Engin) yielded a full-frame sampling frequency of $5 \mathrm{~Hz}$. Furthermore, illumination intensity was adjusted to avoid under or over saturated spots of the brain with an exposure time of $30 \mathrm{~ms}$. A charge-coupled device (CCD) camera (MV-D1024E160-cl, PhotonFocus) with a 12-bit ADC and 1,024 × 1,024 pixels in the image area acquired the images through a macro lens (EF-S $60 \mathrm{~mm} \mathrm{f/2.8,} \mathrm{Macro} \mathrm{USM,} \mathrm{Canon)} \mathrm{over} \mathrm{a} \mathrm{field-of-view} \mathrm{of}$ $(14.7 \mathrm{~mm})^{2}$. Using an aperture of 2.8 , a depth of field of $1.2 \mathrm{~mm}$ was obtained. Image acquisition via a frame grabber (Neon-CLB, Bitflow) was controlled by a custom-made graphical interface developed in MATLAB (The MathWorks, Natick, MA).

\section{Seed-Based Functional Connectivity (fc) Analysis of rs-OIS}

Using the incident light $I_{0}$ and the reflected light $I$ intensities, multispectral optical density $\left(\triangle O D=\log \left(\frac{I_{0}}{I}\right)\right)$ images were converted to $\mathrm{HbO}_{2}$ and $\mathrm{HbR}$ measures $C_{i}(t)$ using the modified Beer-Lambert law and a Moore-Penrose pseudoinverse, according to Delpy et al. (1988):

$$
\Delta O D(\lambda, t)=\sum_{i} \epsilon_{i}(\lambda) C_{i}(t) D(\lambda)
$$

Where the differential path length factor $D(\lambda)$ values were obtained from the literature (Kohl et al., 2000; Dunn et al., 2005) and hemoglobin extinction coefficients $\epsilon_{i}(\lambda)$ were obtained from Prahl (1999). OD values were corrected for the wavelengthdependent response of the CCD sensor and convolved with the LEDs profile (Brieu et al., 2010).

In order to minimize the influence of physiological artifacts on the resting state signal, a general linear model (GLM) (Friston et al., 2006) was used with multiple physiological regressors $X(t)$ : heart rate, respiratory signal, ECG, respiration rate, and the average signal of all those pixels identified as belonging to the cortex:

$$
\beta_{\mathrm{X}}=\mathrm{X}(t)^{+} \mathrm{C}_{\mathrm{i}}(t)
$$

Then the weights $\beta_{X}$ are used to obtain the residual of the regression $C_{i}^{\prime}(t)$, that is then used for the $\mathrm{fc}$ analysis:

$$
\mathrm{C}_{\mathrm{i}}^{\prime}(t)=\mathrm{C}_{\mathrm{i}}(t)-\mathrm{X}(t) \beta_{\mathrm{X}}
$$

$\mathrm{HbO}_{2}$ and $\mathrm{HbR}$ measures, i.e., $\mathrm{C}_{\mathrm{i}}^{\prime}(t)$ were spatially smoothed using an $11 \times 11$ pixels $(\sim 150 \times 150 \mu \mathrm{m})$ gaussian kernel with a 3 pixels $(43 \mu \mathrm{m})$ standard deviation. These chromophore signals were then temporally filtered between 0.009 and 0.08 $\mathrm{Hz}$, using a fourth-order Butterworth filter with zero-phase shift, according to previous fc studies (Guevara et al., 2013c). Images were aligned to a reference atlas through a projective registration using Matlab and anatomical landmarks as the control points for such registration.

Regions of interest, also called seeds in the context of fc, were obtained from previous studies in the rodent brain that have identified a series of cortical regions that exhibit alterations in $\mathrm{fc}$ in several conditions, such as ischemic stroke (Bauer et al., 2014), epilepsy (Guevara et al., 2013b), arterial stiffness (Guevara et al., 2013c), cortical spreading depression (Li et al., 2012), and amyloid- $\beta$ deposition (Bero et al., 2012).

All the seeds were placed using atlas coordinates, using data shown by Jung et al. (2013) as shown in Figure 5A. Time series of every seed were computed as the average value of 17 pixels $(\sim 0.21 \mathrm{~mm})$ around the seed locus. The Pearson correlation values $r$ between each seed time trace were used as the metric for seed-to-seed analysis, converted to Fisher $Z$-values using $Z(r)=\frac{1}{2} \ln \left[\frac{1+r}{1-r}\right]$ before doing group 
level comparisons. Statistical significance was determined by one-sample Wilcoxon rank sum test and $p$-values were false discovery rate (FDR) adjusted (Benjamini and Hochberg, 1995). Effect size was computed with Hedges' g (Hedges and Olkin, 1985). Group-mean connectivity matrices were computed from average seed-to-seed correlation values for both contrasts $\left(\mathrm{HbO}_{2}\right.$ and $\left.\mathrm{HbR}\right)$. Since this model follows a unilateral modification, interhemispheric connectivity values are expected to change, therefore seed-based homotopic connectivity was also investigated as a metric to corroborate those changes, as previous studies (Bero et al., 2012; Guevara et al., 2013a). Seedto-pixel analysis was carried out by computing the functional correlation between each seed time trace and the time course of the pixels marked as belonging to the brain cortex. Fisher $\mathrm{Z}$ transformation was applied to single subject correlation maps, then normalization by subtraction of the mean and division by the standard deviation was performed (Greene et al., 2015). For each normalized seed-based fc map, two-tailed, one sample $t$ test was implemented to characterize the connectivity patterns of each group, assessing its strength relative to zero (Zhan et al., 2014). In order to correct for false positives, a height-threshold of $p<0.05$, FDR-corrected, was implemented (Genovese et al., 2002). Furthermore, clusters with fewer pixels than $5 \%$ of the total number of height-threshold surviving pixels were removed (Warren et al., 2009). In this work we define spatial extent as the ratio of the number of pixels that survived the threshold (FDR-corrected $p<0.05$ in height and $<5 \%$ in extent) to the number of pixels marked as belonging to the brain cortex in our anatomical atlas. Fisher's exact mid-P method was implemented to find out if gender was a confounding factor (Thorvaldsen et al., 2010).

\section{Machine Learning}

Machine learning plays an ever increasingly important role in the neuroimaging field, especially in computer assisted diagnosis (Suzuki et al., 2012). Machine learning techniques may be valuable tools in recognizing connectivity patterns that arise from an inflammatory injury. Therefore, in this paper we apply two different machine learning techniques to assess its value in the context of white matter injury.

\section{Support vector machine}

A support vector machine (SVM) classifier was used to assign labels automatically to rat pups in a blind evaluation, allowing us to explore the research question: Does blind segmentation of subjects using SVM reflect injury status?

Seed-to-seed fc values were chosen as the set of features (56, comprised of both contrasts $\mathrm{HbO}_{2}$ and $\mathrm{HbR}$ ) used as inputs to the SVM classifier depicted in Figure 5B, which separated the data into two groups: $\mathrm{NaCl}$ and LPS. A radial basis function kernel was chosen and sequential minimal optimization was the method used to find the separating hyperplane. Both the box constraint parameter and the kernel sigma were optimized through a grid-search to obtain better accuracy (Gaspar et al., 2012). The performance of the classifier was determined in terms of sensitivity (Se, the proportion of LPS pups identified as such), specificity (Sp, the proportion of control animals correctly identified), positive predictive value (PPV, the proportion of LPS-labeled pups that are actual lesioned animals), negative predictive value (NPV, the ratio of pups labeled as $\mathrm{NaCl}$ that actually belong to the control group), and accuracy (Acc, the proportion of correct labels), which were computed by averaging the results of ten 10 -fold cross-validation runs. In each one of the runs, the data was split into 10 approximately equal partitions, and each in turn was used for training while the remainder is used for validation and testing, i.e., the samples were randomly divided as follows: $70 \%$ for training (9-11 samples), $15 \%$ for validation (1, 2 samples), and $15 \%$ (1, 2 remaining samples) as a completely independent testing, for each run, until all samples were independently tested. A choice of $k=10$ was chosen, as proposed by Borra et al. (Borra and Di Ciaccio, 2010), the number of folds was limited by our sample size.

\section{Artificial neural network}

An artificial neural network (ANN) was designed to answer the following research question: do fc patterns correlate with lesion size? A set of 56 features comprised of the seed-level connectivity matrix of $\mathrm{HbO}_{2}$ and $\mathrm{HbR}$ measures was the input to the ANN as shown on Figure 5D, i.e., the same set of features used in the SVM classifier described above. A two-layer feed-forward ANN with 20 sigmoid hidden neurons and one linear output neuron maps the $\mathrm{fc}$ measures to the lesion size. The number of neurons in the hidden layer was chosen according to the method proposed by Huang (2003). The network was trained using Bayesian regularization, which is more robust to small data sets, as in our case (Burden and Winkler, 2008). Samples from both groups were randomly divided as follows: $70 \%$ for training (7 samples), 15\% for validation (1 sample), and 15\% as a completely independent testing ( 1 sample). The ANN was trained to a maximum of 1,000 epochs and the mean square error goal was assigned to 0.00001 . Accuracy of the ANN was evaluated using correlation coefficient (r) and root-mean square error (RMSEP).

\section{Histology}

Rat pups were perfused through the heart with phosphatebuffered saline solution (PBS) followed by $4 \%$ paraformaldehyde. LPS and sham-injected brains were removed and immersed in paraformaldehyde at $4{ }^{\circ} \mathrm{C}$ for $24 \mathrm{~h}$ and then transferred to $30 \%$ sucrose solution for 2 days prior to cryo-sectioning.

Measurements were performed on coronal rat brain sections, $300 \mu \mathrm{m}$ apart, stained with cresyl violet and scanned with Axioscan Z1 (Carl Zeiss Inc, ON, Canada). Each coronal section (50 $\mu \mathrm{m}$ thick) was thresholded, binarized, and holes were filled in order to delineate the ventricular region using Fiji (Schindelin et al., 2012). Pixels were counted and converted to linear units (228 pixels $/ \mathrm{mm}$ ), the area was multiplied by the slice thickness and all slices were added to find the ventricular volume (Wang et al., 1997). Fractional measurements were performed in order to account for variability in brain size and deformation of histological slices. Due to the fact that ventricles were barely discernable in $\mathrm{NaCl}$ pups, only four of them were analyzed. 


\section{RESULTS}

The optical intrinsic signal obtained from young rat allowed the characterization of resting state homeostasis in normal and inflammatory conditions. Three complementary approaches were used: (i) Seed-based functional connectivity analysis of rsOIS; (ii) Support vector machine; (iii) Artificial Neural Network.

\section{Seed-Based Functional Connectivity Analysis of rs-OIS}

Connectivity matrices for both groups and both contrasts $\left(\mathrm{HbO}_{2}\right.$ and $\mathrm{HbR}$ ) were evaluated (Figure 1). When identifying seed based functional connectivity analysis of rs-OIS in sham animals, connectivity matrices were better identified in $\mathrm{HbR}$ contrast (Figure 1).

The association between gender and $\mathrm{fc}$ measures was not statistically significant $(p=0.7552)$. As displayed on the connectivity matrices, our results show that homotopic fc values in the motor cortex and cingulate region are impaired in the injured group; for $\mathrm{HbR}$ contrast homotopic connectivity in retrosplenial region is decreased as well. Positive connections are less numerous in the LPS group, and this diminution is more remarkable in HbR connectivity matrices, as shown in Figure 1D. This decrease of $\mathrm{fc}$ was also present in the anticorrelated edges. Yet, there were no statistical differences in seedto-seed connectivity between the groups, after FDR correction (data not shown).

\section{Spatial Connectivity Extent}

To further characterize the impact of cerebral inflammation in the developing brain we studied the spatial extent of networks. We generated group-averaged fc maps for $\mathrm{HbR}$ and $\mathrm{HbO}_{2}$ contrasts with seeds placed in the motor cortex, the cingulate cortex, the somatosensory cortex and the retrosplenial cortex (Figures 2, 3). Inflammation caused a significant decrease of spatial extent across cortical regions, when compared to the $\mathrm{NaCl}$ group (LPS $=1.5 \pm 1 \%$ vs. $\mathrm{NaCl}=45.9 \pm 0.6 \%$, ${ }^{* *} p=0.0036$, Hedges' $g=1.0861$, as shown in Figure 4A). A similar reduction is observed in $\mathrm{HbO}_{2} \mathrm{fc}$ maps displayed in Figure 3 (LPS $=1.1 \pm 0.6 \%$ vs. $\mathrm{NaCl}=12.7 \pm 4.1 \%$,

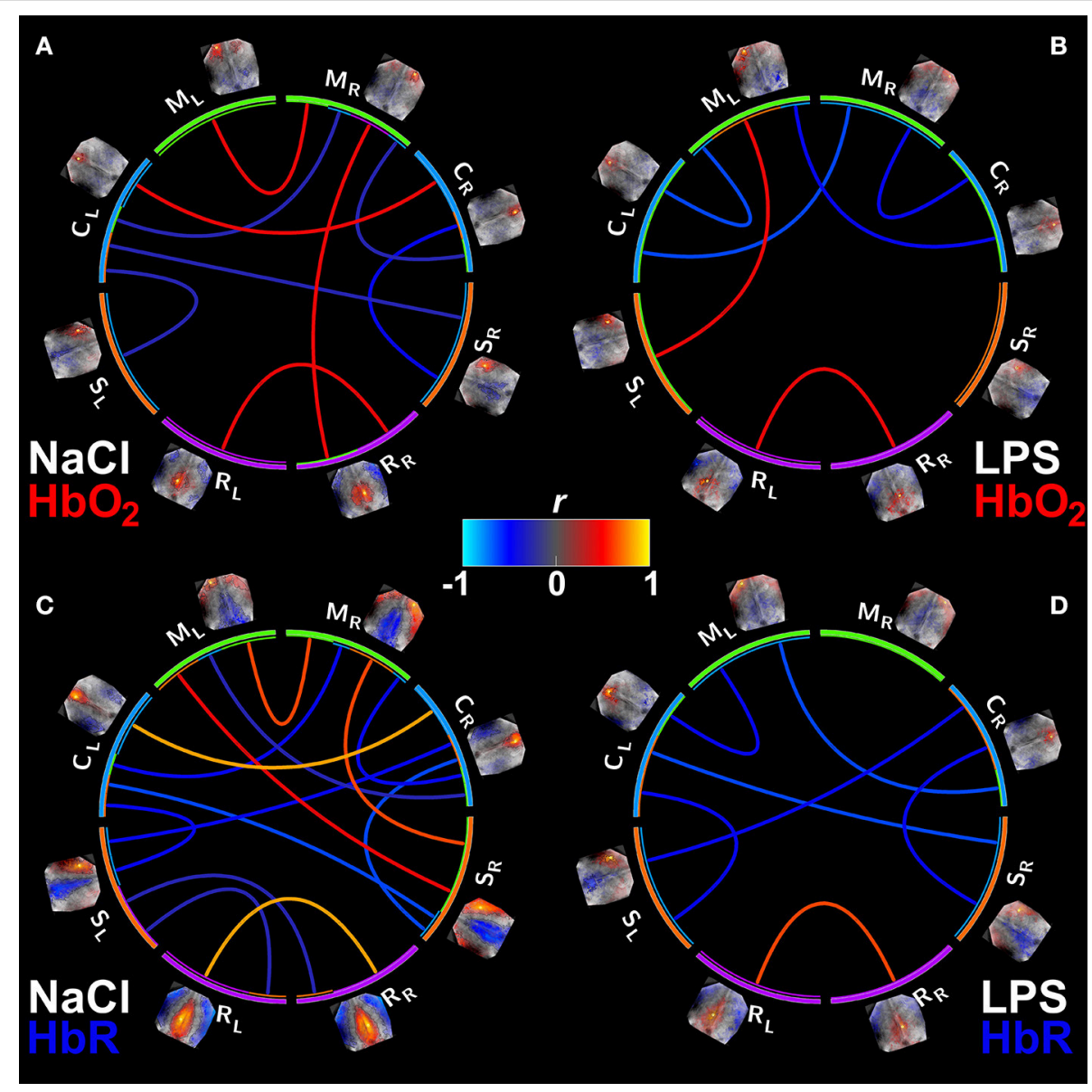

FIGURE 1 | Group mean connectivity matrices visualized with Circos (Krzywinski et al., 2009). (A,B) Show the connectivity matrices for $\mathrm{HbO}_{2}$ contrast from the NaCl and LPS groups, respectively. (C,D) Same as (A,B) but for HbR. Supra-threshold edges are shown (FDR-corrected $p<0.05)$. M, motor cortex; C, cingulate cortex; S, somatosensory cortex; $R$, retrosplenial cortex. Subscripts $L$ and $R$ indicate hemisphere side. Note the significant reduction in connectivity in animals exposed to inflammation with a larger effect on $\mathrm{HbR}$ contrast. 


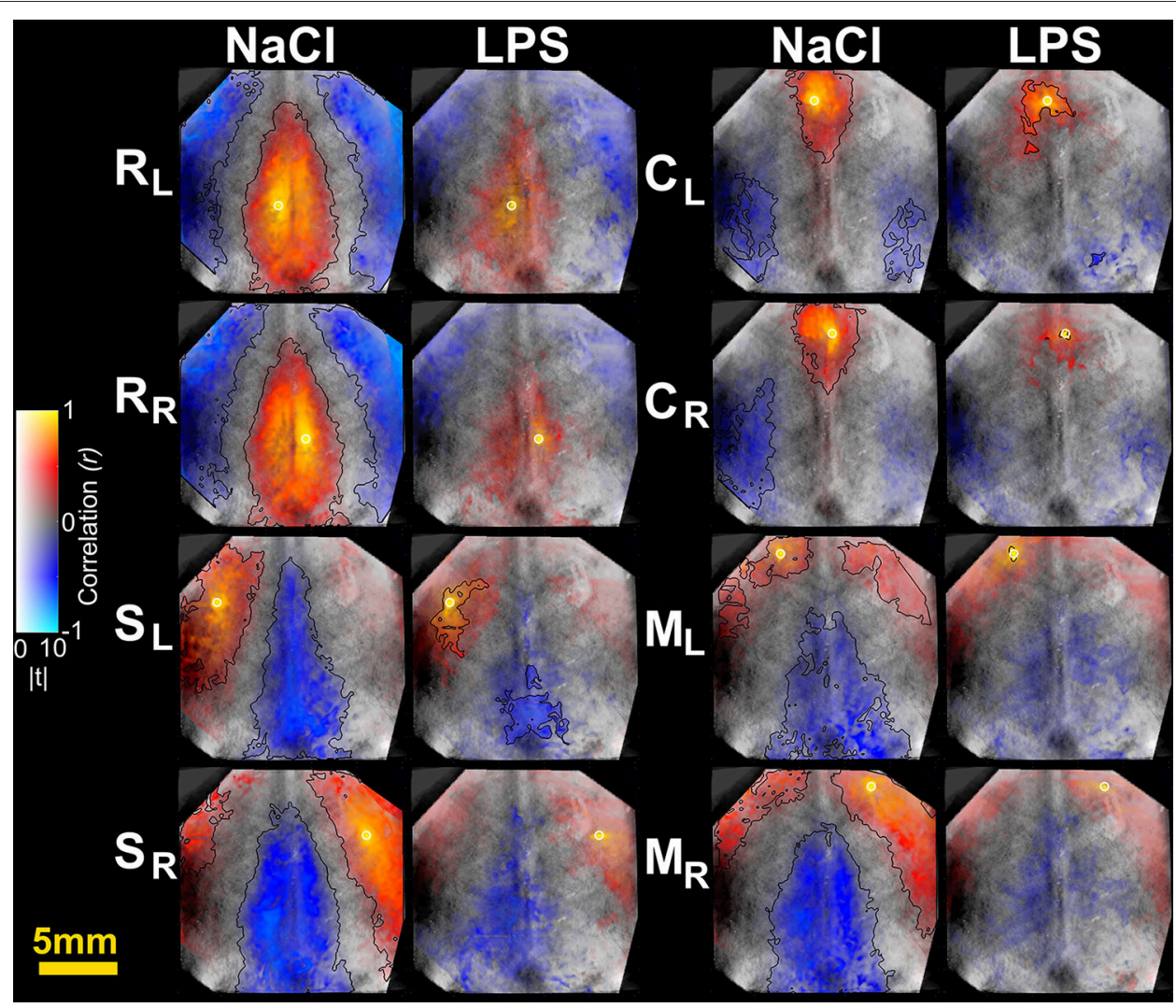

FIGURE 2 | Seed-based correlation maps for HbR contrast. T-statistics are encoded as transparency, while the correlation values are encoded as hue values (Allen et al., 2012). A height-threshold of $p<0.05$, FDR-corrected, was implemented. Clusters with fewer pixels than $5 \%$ of the total number of suprathreshold pixels were removed (Warren et al., 2009). A black contour is shown for FDR-corrected $p<0.05$ and spatial extent threshold $>5 \%$. Note the decrease of significantly correlated pixels in the LPS group. M, motor cortex; C, cingulate cortex; S, somatosensory cortex; R, retrosplenial cortex. Subscripts L and R indicate hemisphere.

${ }^{*} p=0.0452$, Hedges' $g=0.9338$, as shown in Figure 4A). A large effect size was detected in both contrasts. Moreover, this decrease is consistent across seed locations and intrinsic contrasts, as shown in Figures 4B,C. These results indicate decreased functional connectivity following white matter injury across different regions and contrasts.

\section{Machine Learning Analysis of rs-OIS Using Support Vector Machine to Identify Injury}

Separating the data into two groups: sham animals and injured ones, we built a confusion matrix for the SVM binary classifier using the set of $\mathrm{HbO}_{2}+\mathrm{HbR}$ features (Figure 5B), following the cross-validation procedure mentioned in the Methods Section. Only one subject from LPS group was incorrectly classified as belonging to $\mathrm{NaCl}$ group. This resulted in a $92.3 \%$ accuracy (Figure 5C). Other kernels, such as a linear kernel did not yield higher accuracy and therefore are not presented in this work.

\section{Machine Learning Analysis of rs-OIS Using an Artificial Neural Network to Quantify Injury}

The implementation of an ANN model (Figure 5D) enabled the prediction of fractional lesion volume based on fc-OIS measures. In similar fashion to SVM classifier described above, the connectivity matrix comprised of both $\mathrm{HbO}_{2}$ and $\mathrm{HbR}$ contrast, totaling 56 seed-to-seed connectivity values, was used as input to our ANN. Using Bayesian regularization as our training algorithm, we found a good agreement between the results predicted by this model and the degree of ventricular dilatation, (regression coefficient $r=0.9431, p=0.0020$, and a RMSEP $=$ 2.25\%; Figure 5E). Other back-propagation algorithms such as Levenberg-Marquardt training failed to predict injury accurately (results not shown). The number of neurons in the hidden layer was chosen as a compromise in prediction error and computation time. An increase in the number of neurons did not result in better performance, and architectures with fewer neurons did not yield accuracy $>90 \%$, thus 20 neurons was a justifiable choice.

\section{DISCUSSION}

Optical intrinsic signal represents a major advantage over fcMRI due to a very high temporal and spatial resolution. It is straightforward to setup compared to MRI, with neither associated mechanical vibrations nor loud sounds, thus avoiding vibration and acoustic noise-related artifacts; while providing better access to the animal, thus facilitating its manipulation. It is 


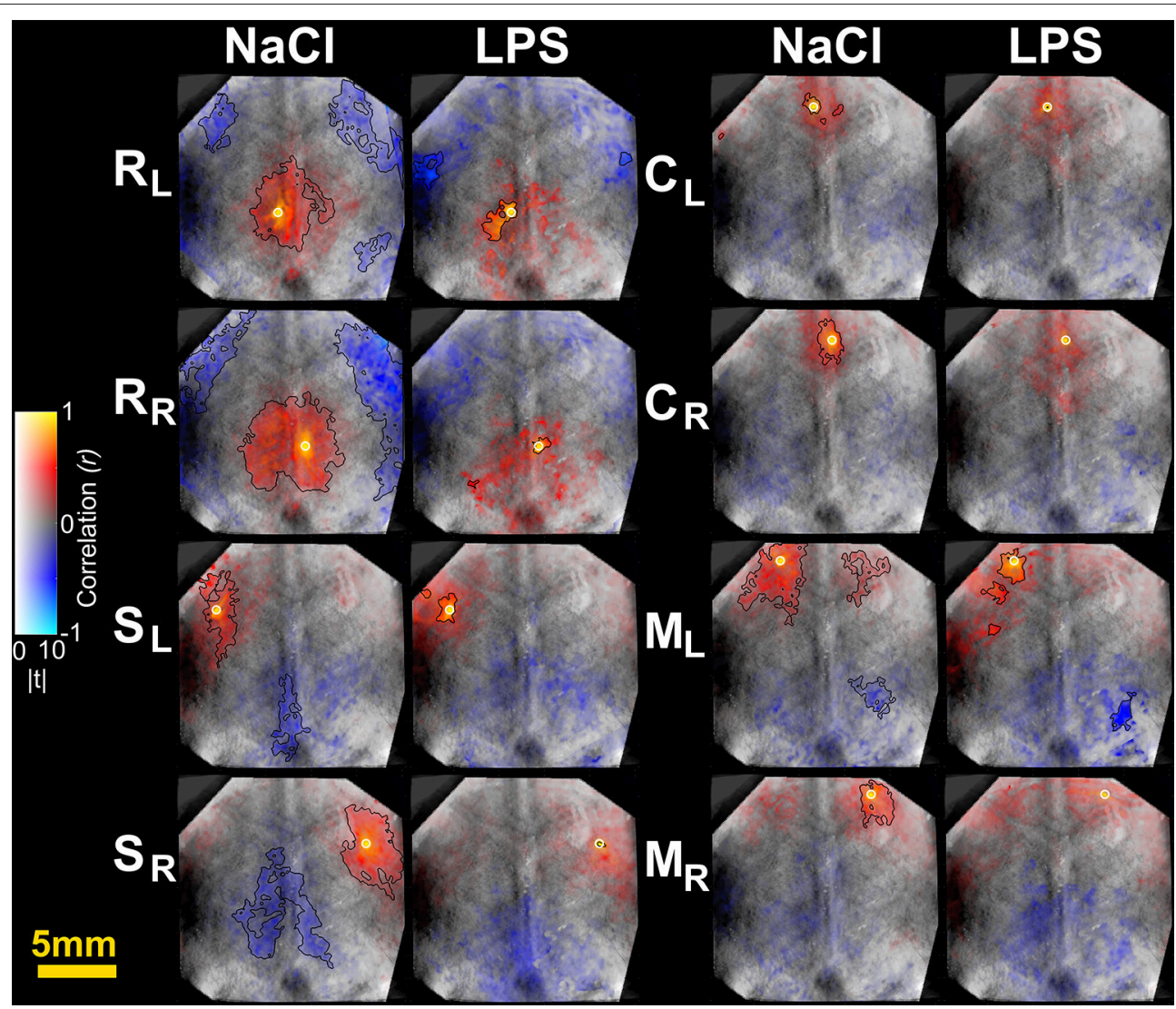

FIGURE 3 | Seed-based correlation maps for $\mathrm{HbO}_{2}$ contrast. T-statistics are encoded as transparency, while the correlation values are encoded as hue values (Allen et al., 2012). A height-threshold of $p<0.05$, FDR-corrected, was implemented. Clusters with fewer pixels than $5 \%$ of the total number of suprathreshold pixels were removed (Warren et al., 2009). A black contour is shown for FDR-corrected $p<0.05$ and spatial extent threshold $>5 \%$. Note the decrease of significantly correlated pixels in the LPS group. M, motor cortex; C, cingulate cortex; S, somatosensory cortex; R, retrosplenial cortex. Subscripts L and R indicate hemisphere.
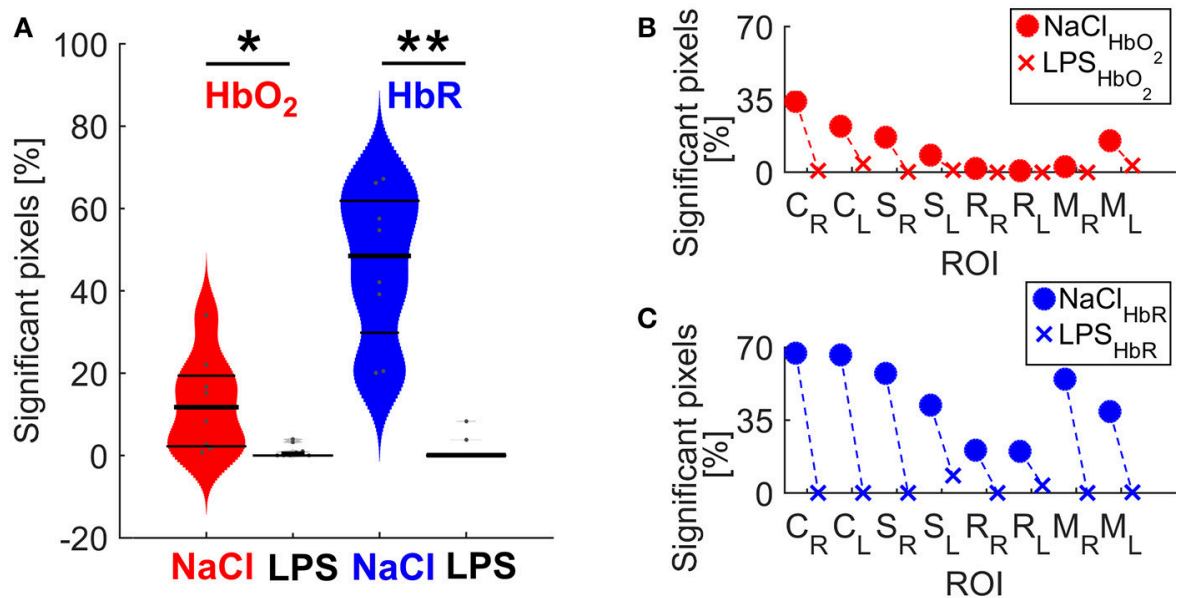

FIGURE 4 | (A) Spatial extent differences between $\mathrm{NaCl}$ and LPS groups. The shape of each distribution is plotted, overlaid with data points and lines denoting 25 , 50 , and 75 percentiles. ${ }^{\star} P=0.0452$ and Hedges' $g=0.9338$ for $\mathrm{HbO}_{2}$; ${ }^{\star \star} p=0.0036$ and Hedges' $g=1.0861$ for HbR. (B) Spatial extent by individual networks for $\mathrm{HbO}_{2}$ contrast (C) Spatial extent by individual networks for HbR contrast. M, motor cortex; C, cingulate cortex; $\mathrm{S}$, somatosensory cortex; R, retrosplenial cortex. Subscripts $L$ and $R$ indicate hemisphere. 

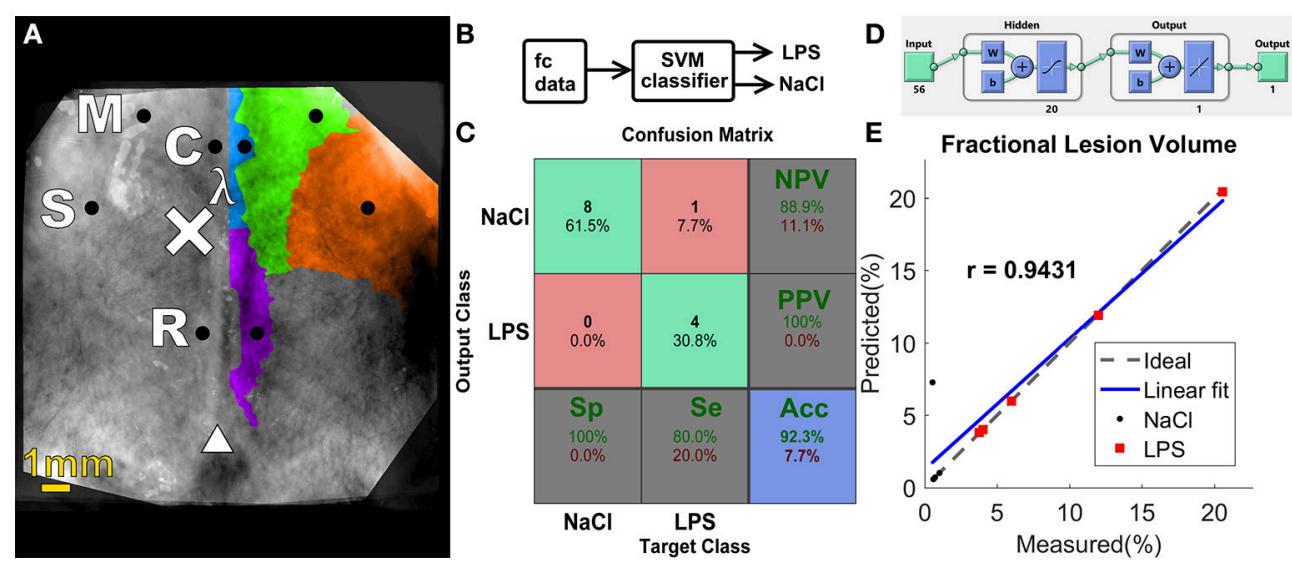

FIGURE 5 | (A) Functional regions on the rat cortex, seed placement and size, manually constructed from data shown by Jung et al. (2013). Black circles indicate seed position and size. Injection site is denoted by the " $\times$ " symbol, lambda denoted by $\lambda$ and bregma indicated by triangle symbol. M, motor; C, cingulate; S, somatosensory; R, retrosplenial. (B) Block diagram of the proposed classifier (C) Confusion matrix for the SVM classifier. (D) Graphical diagram of the ANN. (E) Regression plot for ANN predicted ventricular size vs. measured ventricular size.

obviously restricted to $2 \mathrm{D}$ mapping and not a technique of choice for deep brain structures. This is an important limitation when performing regression on rs-OIS data; there is the possibility that global signal regression leads to spurious negative correlations (Murphy et al., 2009; Saad et al., 2012), although some works suggest a physiological basis for anticorrelated networks (Fox et al., 2009). Unfortunately, a viable alternate option is not provided by the OIS technique. Further work is needed on this issue.

As shown in adult mice (Guevara et al., 2013c) we found also that $\mathrm{HbR}$ contrast appears to be more sensitive to uncover resting state networks in the young rat brain (Figure 1). Interestingly $\mathrm{HbR}$ has been shown to closely match the BOLD contrast in fMRI experiments (Huppert et al., 2006).

When compared to adult mice (Guevara et al., 2013c), rsOIS in young rats detected similar functional correlation maps, across different seeds, showing high degree of inter-hemispheric symmetry in control animals.

In the developing rat brain, an inflammatory brain injury was found to cause significant long standing disruption of resting state activity using rs-OIS with a reduction of connectivity in several networks particularly on motor and cingulate networks. The clear impact of inflammation on these networks is not unexpected considering the site of LPS injection. Nevertheless, the identification of persisting deficits is remarkable as these results are in agreement with preterm infants with substantial white matter injury, with a significant reduction on motor networks and in the vicinity of clear injury (Smyser et al., 2013).

When studying averaged seed-based correlation maps, inflammation was shown to cause a significant decrease of spatial extent across cortical regions, that was highly significant on HbR maps (Figures 2-4). This difference in HbR with respect to $\mathrm{HbO}_{2}$ may be explained by the higher contribution of $\mathrm{HbR}$ to absorption at 590 and $630 \mathrm{~nm}$ wavelengths. As mentioned in the introduction similar reduction of correlated voxels where found in preterm infants when compared to term infants imaged at term equivalent age (Smyser et al., 2010). Translating this approach on pre-clinical fcMRI in young developing rat or mice brains might prove difficult as partial volume effect might dampen differences between groups especially in small correlated regions such as in the cingulate cortex, where fc-OIS was able to find a difference of $\sim 0.6 \mathrm{~mm}^{2}$. This small, albeit significant difference might be challenging to unravel using fcMRI in rodents, where voxel size is usually between $0.5 \mathrm{~mm}^{3}$ (Liang et al., 2012) and $1 \mathrm{~mm}^{3}$ (Harris et al., 2015).

The use of a SVM allowed us despite the limited sample size, to efficiently classify injured from intact developing brains with accuracy above $90 \%$. In contrast to seed based analysis it provides a categorical result with no indication regarding the type of changes. The advantages and limitations still need to be properly addressed especially if used to determine therapeutic efficiency. Nevertheless, it is a first step in detecting injury through optical imaging, since SVM was shown to identify injury with high accuracy, using rs-OIS data. Similarly, Vergara et al. have used SVM with a leave-one-out cross validation to classify mild traumatic brain injury based on fcMRI with an accuracy of $84.1 \%$ that was superior to DTI analysis (Vergara et al., 2017). This approach was also successfully applied in a cohort of preterm infants also using fcMRI with an $84 \%$ accuracy and a specificity of $78 \%$ (Smyser et al., 2016a). The range of accuracy probably depends on the severity of injury. It is noteworthy that this approach remains extremely efficient in the setting of mild traumatic brain injury and in a cohort of healthy preterm infants at term with no overt brain injury. The increased temporal resolution of rs-OIS over fcMRI may increase the quality of resting state assessment. It is possible that in young rodent, rs-OIS might reveal itself superior in comparative studies with fcMRI.

The use of an ANN allowed us to assess the degree of injury with a highly significant correlation with the degree of ventricular 
dilation. Machine learning approaches have proven useful in neonatal populations, e.g., using SVM to predict gestational age (Smyser et al., 2016a), ANN to assess mortality risk (Zernikow et al., 1998) or heuristic methods to detect seizures (Ansari et al., 2016). One limitation of ANN, and machine learning in general, is the fact that the only observed states are the inputs and the outputs, making interpretation of their inner functions exceedingly difficult (Castelvecchi, 2016). Considering the small number of animals, rs-OIS appears to be a highly reliable tool, sensitive enough to pick up differences in lesion load.

\section{CONCLUSIONS}

An analysis of resting state networks in the developing rodent brain together with the impact of inflammatory white matter injury was presented using fc measurements and machine learning techniques. Our approach was based on creating group-level fc statistical maps where a significant decrease in connectivity was observed in the case of the injured group; furthermore, seed-based $\mathrm{fc}$ measures were analyzed through SVM to identify connectivity patterns differences between $\mathrm{NaCl}$ and LPS groups. Those same $\mathrm{fc}$ measures were fed to an ANN that allowed the prediction of fractional lesion volume. Different fc approaches (ICA, graph theory, clustering, etc.) and alternatives to the chosen machine learning algorithms need further investigation on the basis of a larger sample. Another drawback of this study is the assumption of stationarity in fc, thus requiring the consideration of dynamic models in future studies (Hansen et al., 2015). Nevertheless, the translation of both

\section{REFERENCES}

Aertsen, A. M., Gerstein, G. L., Habib, M. K., and Palm, G. (1989). Dynamics of neuronal firing correlation: modulation of "effective connectivity". J. Neurophysiol. 61, 900-917.

Allen, E. A., Erhardt, E. B., and Calhoun, V. D. (2012). Data visualization in the neurosciences: overcoming the curse of dimensionality. Neuron 74, 603-608. doi: 10.1016/j.neuron.2012.05.001

Ansari, A. H., Cherian, P. J., Dereymaeker, A., Matic, V., Jansen, K., De Wispelaere, L., et al. (2016). Improved multi-stage neonatal seizure detection using a heuristic classifier and a data-driven post-processor. Clin. Neurophysiol. 127, 3014-3024. doi: 10.1016/j.clinph.2016.06.018

Bauer, A. Q., Kraft, A. W., Wright, P. W., Snyder, A. Z., Lee, J.-M., and Culver, J. P. (2014). Optical imaging of disrupted functional connectivity following ischemic stroke in mice. Neuroimage 99, 388-401. doi: 10.1016/j.neuroimage.2014.05.051

Bélanger, S., de Souza, B. O. F., Casanova, C., and Lesage, F. (2016). Correlation of hemodynamic and fluorescence signals under resting state conditions in mice's barrel field cortex. Neurosci. Lett. 616, 177-181. doi: 10.1016/j.neulet.2016.01.067

Belliveau, J. W., Kennedy, D. N., McKinstry, C., Buchbinder, B. R., Weisskoff, R. M., Cohen, M. S., et al. (1991). Functional mapping of the human visual cortex by magnetic resonance imaging. Science 254, 716-719.

Benjamini, Y., and Hochberg, Y. (1995). Controlling the false discovery rate: a practical and powerful approach to multiple testing. J. R. Stat. Soc. B 57, 289-300.

Benveniste, H., and Blackband, S. (2002). MR microscopy and high resolution small animal MRI: applications in neuroscience research. Prog. Neurobiol. 67, 393-420. doi: 10.1016/S0301-0082(02)00020-5

Bero, A. W., Bauer, A. Q., Stewart, F. R., White, B. R., Cirrito, J. R., Raichle, M. E., et al. (2012). Bidirectional relationship between functional connectivity machine-learning approaches, to a bedside optical monitoring system, such as fNIRS, could be helpful in providing early diagnosis in preterm infants especially when evaluating small cohorts.

\section{AUTHOR CONTRIBUTIONS}

EG, FL, and GL conceived and designed the experiments; WP, CT, LA, IL, and GL performed the experiments; EG, CT, LA, IL, and GL analyzed the data; FL and GL contributed materials and analysis tools; EG, CT, FL, and GL wrote the paper.

\section{FUNDING}

This work was supported by a grant from the Canadian Institutes of Health Research-Institute of Human Development, Child and Youth Health (IHDCYH) \# 136908 and in part (EG and GL) by XVe Groupe de travail Québec-Mexique 2015-2016, by the Québec Bio-Imaging Network and by the "Cátedras CONACYT" program, project 528 (EG). GL is supported by a start-up grant from the Research Center of Sainte-Justine and partly by salary award from Fonds de recherche du Québec-Santé. FL is supported by a NSERC Discovery and CIHR Grant.

\section{ACKNOWLEDGMENTS}

The authors cordially thank Dr. Samuel Bélanger for advice on OIS imaging and Dr. Elena Allen for advice on data visualization.

and amyloid- $\beta$ deposition in mouse brain. J. Neurosci. 32, 4334-4340. doi: 10.1523/JNEUROSCI.5845-11.2012

Biswal, B., Yetkin, F. Z., Haughton, V. M., and Hyde, J. S. (1995). Functional connectivity in the motor cortex of resting human brain using echo-planar MRI. Magn. Reson. Med. 34, 537-541. doi: 10.1002/mrm.1910340409

Borra, S., and Di Ciaccio, A. (2010). Measuring the prediction error. A comparison of cross-validation, bootstrap and covariance penalty methods. Comput. Stat. Data Analysis 54, 2976-2989. doi: 10.1016/j.csda.2010.03.004

Brieu, N., Beaumont, E., Dubeau, S., Cohen-Adad, J., and Lesage, F. (2010). Characterization of the hemodynamic response in the rat lumbar spinal cord using intrinsic optical imaging and laser speckle. J. Neurosci. Methods 191, 151-157. doi: 10.1016/j.jneumeth.2010.06.012

Burden, F., and Winkler, D. (2008). Bayesian regularization of neural networks. Methods Mol. Biol. 458, 25-44. doi: 10.1007/978-1-60327-101-1_3

Buxton, R. B. (2013). The physics of functional magnetic resonance imaging (fMRI). Rep. Prog. Phys. 76:096601. doi: 10.1088/0034-4885/76/9/096601

Cai, Z., Pang, Y., Lin, S., and Rhodes, P. G. (2003). Differential roles of tumor necrosis factor-alpha and interleukin-1 beta in lipopolysaccharideinduced brain injury in the neonatal rat. Brain Res. 975, 37-47. doi: 10.1016/S0006-8993(03)02545-9

Cang, J., Kalatsky, V. A., Löwel, S., and Stryker, M. P. (2005). Optical imaging of the intrinsic signal as a measure of cortical plasticity in the mouse. Vis. Neurosci. 22, 685-691. doi: 10.1017/S0952523805225178

Castelvecchi, D. (2016). Can we open the black box of AI? Nature 538, 20-23. doi: $10.1038 / 538020 \mathrm{a}$

Chen, M., Xia, D., Min, C., Zhao, X., Chen, Y., Liu, L., et al. (2016). Neonatal repetitive pain in rats leads to impaired spatial learning and dysregulated hypothalamic-pituitary-adrenal axis function in later life. Sci. Rep. 6:39159. doi: 10.1038/srep39159

Delpy, D. T., Cope, M., van der Zee, P., Arridge, S., Wray, S., and Wyatt, J. (1988). Estimation of optical pathlength through tissue from 
direct time of flight measurement. Phys. Med. Biol. 33, 1433-1442. doi: 10.1088/0031-9155/33/12/008

Doria, V., Beckmann, C. F., Arichi, T., Merchant, N., Groppo, M., Turkheimer, F. E., et al. (2010). Emergence of resting state networks in the preterm human brain. Proc. Natl. Acad. Sci. U.S.A. 107, 20015-20020. doi: $10.1073 /$ pnas. 1007921107

Dunn, A. K., Devor, A., Dale, A. M., and Boas, D. A. (2005). Spatial extent of oxygen metabolism and hemodynamic changes during functional activation of the rat somatosensory cortex. Neuroimage 27, 279-290. doi: 10.1016/j.neuroimage.2005.04.024

Fan, L., Pang, Y., Lin, S., and Tien, L. (2005). Minocycline reduces lipopolysaccharide-induced neurological dysfunction and brain injury in the neonatal rat. J. Neurosci. Res. 82, 71-82. doi: 10.1002/jnr.20623

Farrant, K., and Uddin, L. Q. (2016). Atypical developmental of dorsal and ventral attention networks in autism. Dev. Sci. 19, 550-563. doi: 10.1111/desc.12359

Fox, M. D., Zhang, D., Snyder, A. Z., and Raichle, M. E. (2009). The global signal and observed anticorrelated resting state brain networks. J. Neurophysiol. 101, 3270-3283. doi: 10.1152/jn.90777.2008

Friston, K. J., Ashburner, J. T., Kiebel, S. J., Nichols, T. E., and Penny, W. D. (2006). Statistical Parametric Mapping: The Analysis of Functional Brain Images, 1st Edn. Great Britain: Academic Press.

Friston, K. J., Frith, C. D., Liddle, P. F., and Frackowiak, R. S. (1993). Functional connectivity: the principal-component analysis of large (PET) data sets. J. Cereb. Blood Flow Metab. 13, 5-14. doi: 10.1038/jcbfm.1993.4

Frostig, R. D., Lieke, E. E., Ts'o, D. Y., and Grinvald, A. (1990). Cortical functional architecture and local coupling between neuronal activity and the microcirculation revealed by in vivo high-resolution optical imaging of intrinsic signals. Proc. Natl. Acad. Sci. U.S.A. 87, 6082-6086. doi: $10.1073 /$ pnas.87.16.6082

Gaspar, P., Carbonell, J., and Oliveira, J. L. (2012). On the parameter optimization of Support Vector Machines for binary classification. J. Integr. Bioinform. 9:201. doi: 10.2390/biecoll-jib-2012-201

Genovese, C. R., Lazar, N. A., and Nichols, T. (2002). Thresholding of statistical maps in functional neuroimaging using the false discovery rate. Neuroimage 15, 870-878. doi: 10.1006/nimg.2001.1037

Gili, T., Cercignani, M., Serra, L., Perri, R., Giove, F., Maraviglia, B., et al. (2011). Regional brain atrophy and functional disconnection across Alzheimer's disease evolution. J. Neurol. Neurosurg. Psychiatr. 82, 58-66. doi: 10.1136/jnnp.2009.199935

Greene, C. S., Krishnan, A., Wong, A. K., Ricciotti, E., Zelaya, R. A., Himmelstein, D. S., et al. (2015). Understanding multicellular function and disease with human tissue-specific networks. Nat. Genet. 47, 569-576. doi: 10.1038/ng.3259

Greicius, M. D., Srivastava, G., Reiss, A. L., and Menon, V. (2004). Defaultmode network activity distinguishes Alzheimer's disease from healthy aging: evidence from functional MRI. Proc. Natl. Acad. Sci. U.S.A. 101, 4637-4642. doi: 10.1073/pnas.0308627101

Grinvald, A., Lieke, E., Frostig, R. D., Gilbert, C. D., and Wiesel, T. N. (1986). Functional architecture of cortex revealed by optical imaging of intrinsic signals. Nature 324, 361-364. doi: 10.1038/324361a0

Guevara, E., Berti, R., Londono, I., Xie, N., Bellec, P., Lesage, F., et al. (2013a). Imaging of an Inflammatory Injury in the Newborn Rat Brain with Photoacoustic Tomography. PLOS ONE 8:e83045. doi: 10.1371/journal.pone.0083045

Guevara, E., Pouliot, P., Nguyen, D. K., and Lesage, F. (2013b). Optical imaging of acute epileptic networks in mice. J. Biomed. Opt. 18:076021. doi: 10.1117/1.JBO.18.7.076021

Guevara, E., Sadekova, N., Girouard, H., and Lesage, F. (2013c). Optical imaging of resting-state functional connectivity in a novel arterial stiffness model. Biomed. Opt. Express 4, 2332-2346. doi: 10.1364/BOE.4.002332

Hansen, E. C. A., Battaglia, D., Spiegler, A., Deco, G., and Jirsa, V. K. (2015). Functional connectivity dynamics: modeling the switching behavior of the resting state. Neuroimage 105, 525-535. doi: 10.1016/j.neuroimage.2014.11.001

Harris, A. P., Lennen, R. J., Marshall, I., Jansen, M. A., Pernet, C. R., Brydges, N. M., et al. (2015). Imaging learned fear circuitry in awake mice using fMRI. Eur. J. Neurosci. 42, 2125-2134. doi: 10.1111/ejn.12939

He, B., and Liu, Z. (2008). Multimodal functional neuroimaging: integrating functional MRI and EEG/MEG. IEEE Rev. Biomed. Eng. 1, 23-40. doi: 10.1109/RBME.2008.2008233

He, L., and Parikh, N. A. (2016). Brain functional network connectivity development in very preterm infants: the first six months.
Early Hum. Dev. 98, 29-35. doi: 10.1016/j.earlhumdev.2016. 06.002

Hedges, L. V., and Olkin, I. (1985). Statistical Methods for Meta-Analysis. Orlando, FL: Academic Press.

Hillman, E. M. (2007). Optical brain imaging in vivo: techniques and applications from animal to man. J. Biomed. Opt. 12, 051402. doi: 10.1117/1.2789693

Huang, G.-B. (2003). Learning capability and storage capacity of twohidden-layer feedforward networks. IEEE Trans. Neural Netw. 14, 274-281. doi: 10.1109/TNN.2003.809401

Huppert, T. J., Hoge, R. D., Dale, A. M., Franceschini, M. A., and Boas, D. A. (2006). Quantitative spatial comparison of diffuse optical imaging with blood oxygen level-dependent and arterial spin labeling-based functional magnetic resonance imaging. J. Biomed. Opt. 11, 064018. doi: 10.1117/1.2400910

Huttunen, J. K., Gröhn, O., and Penttonen, M. (2008). Coupling between simultaneously recorded BOLD response and neuronal activity in the rat somatosensory cortex. Neuroimage 39, 775-785. doi: 10.1016/j.neuroimage.2007.06.042

Jung, J., Kwak, B. K., Reddy, A. M., Ha, B. C., Shim, H. J., Byun, J. S., et al. (2013). Characterization of photothrombotic cerebral infarction model at sensorimotor area of functional map in rat. J. Neurol. Sci. 30, 617-628.

Jonckers, E., Shah, D., Hamaide, J., Verhoye, M., and Van der Linden, A. (2015). The power of using functional fMRI on small rodents to study brain pharmacology and disease. Front. Pharmacol. 6:231. doi: 10.3389/fphar.2015.00231

Jonckers, E., Van Audekerke, J., De Visscher, G., Van der Linden, A., and Verhoye, M. (2011). Functional connectivity fMRI of the rodent brain: comparison of functional connectivity networks in rat and mouse. PLOS ONE 6:e18876. doi: 10.1371/journal.pone.0018876

Kohl, M., Lindauer, U., Royl, G., Kuhl, M., Gold, L., Villringer, A., et al. (2000). Physical model for the spectroscopic analysis of cortical intrinsic optical signals. Phys. Med. Biol. 45, 3749-3764. doi: 10.1088/0031-9155/45/12/317

Kozberg, M. G., Chen, B. R., DeLeo, S. E., Bouchard, M. B., and Hillman, E. M. C. (2013). Resolving the transition from negative to positive blood oxygen leveldependent responses in the developing brain. Proc. Natl. Acad. Sci. U.S.A. 110, 4380-4385. doi: 10.1073/pnas.1212785110

Krzywinski, M., Schein, J., Birol, I., Connors, J., Gascoyne, R., Horsman, D., et al. (2009). Circos: an information aesthetic for comparative genomics. Genome Res. 19, 1639-1645. doi: 10.1101/gr.092759.109

Lee, W., Morgan, B. R., Shroff, M. M., Sled, J. G., and Taylor, M. J. (2013). The development of regional functional connectivity in preterm infants into early childhood. Neuroradiology 55(Suppl. 2), 105-111. doi: 10.1007/s00234-013-1232-z

Lewis, C. M., Baldassarre, A., Committeri, G., Romani, G. L., and Corbetta, M. (2009). Learning sculpts the spontaneous activity of the resting human brain. Proc. Natl. Acad. Sci. U.S.A. 106, 17558-17563. doi: 10.1073/pnas.0902455106

Li, B., Zhou, F., Luo, Q., and Li, P. (2012). Altered resting-state functional connectivity after cortical spreading depression in mice. Neuroimage 63, 1171-1177. doi: 10.1016/j.neuroimage.2012.08.024

Liang, Z., King, J., and Zhang, N. (2012). Intrinsic organization of the anesthetized brain. J. Neurosci. 32, 10183-10191. doi: 10.1523/JNEUROSCI.1020-12.2012

Lodygensky, G. A., Kunz, N., Perroud, E., Somm, E., Mlynarik, V., Hüppi, P. S., et al. (2014). Definition and quantification of acute inflammatory white matter injury in the immature brain by MRI/MRS at high magnetic field. Pediatr. Res. 75, 415-423. doi: 10.1038/pr.2013.242

Lodygensky, G. A., West, T., Stump, M., Holtzman, D. M., Inder, T. E., and Neil, J. J. (2010). In vivo MRI analysis of an inflammatory injury in the developing brain. Brain Behav. Immun. 24, 759-767. doi: 10.1016/j.bbi.2009.11.005

Mankinen, K., Jalovaara, P., Paakki, J.-J., Harila, M., Rytky, S., Tervonen, O., et al. (2012). Connectivity disruptions in resting-state functional brain networks in children with temporal lobe epilepsy. Epilepsy Res. 100, 168-178. doi: 10.1016/j.eplepsyres.2012.02.010

Mevel, K., and Fransson, P. (2016). The functional brain connectome of the child and autism spectrum disorders. Acta Paediatr. 105, 1024-1035. doi: 10.1111/apa.13484

Mulders, P. C., van Eijndhoven, P. F., Schene, A. H., Beckmann, C. F., and Tendolkar, I. (2015). Resting-state functional connectivity in major depressive disorder: a review. Neurosci. Biobehav. Rev. 56, 330-344. doi: 10.1016/j.neubiorev.2015.07.014

Murphy, K., Birn, R. M., Handwerker, D. A., Jones, T. B., and Bandettini, P. A. (2009). The impact of global signal regression on resting state 
correlations: are anti-correlated networks introduced? Neuroimage 44, 893-905. doi: 10.1016/j.neuroimage.2008.09.036

Ogawa, S., and Lee, T. M. (1990). Magnetic resonance imaging of blood vessels at high fields: in vivo and in vitro measurements and image simulation. Magn. Reson. Med. 16, 9-18. doi: 10.1002/mrm.1910160103

Ogawa, S., Lee, T. M., Nayak, A. S., and Glynn, P. (1990). Oxygenation-sensitive contrast in magnetic resonance image of rodent brain at high magnetic fields. Magn. Reson. Med. 14, 68-78. doi: 10.1002/mrm.1910140108

Paasonen, J., Salo, R. A., Shatillo, A., Forsberg, M. M., Närväinen, J., Huttunen, J. K., et al. (2016). Comparison of seven different anesthesia protocols for nicotine pharmacologic magnetic resonance imaging in rat. Eur. Neuropsychopharmacol. 26, 518-531. doi: 10.1016/j.euroneuro.2015. 12.034

Pan, W.-J., Billings, J. C. W., Grooms, J. K., Shakil, S., and Keilholz, S. D. (2015). Considerations for resting state functional MRI and functional connectivity studies in rodents. Front. Neurosci. 9:269. doi: 10.3389/fnins.2015.00269

Pang, Y., Cai, Z., and Rhodes, P. G. (2003). Disturbance of oligodendrocyte development, hypomyelination and white matter injury in the neonatal rat brain after intracerebral injection of lipopolysaccharide. Brain Res. Dev. Brain Res. 140, 205-214. doi: 10.1016/S0165-3806(02)00606-5

Peraza, L. R., Colloby, S. J., Firbank, M. J., Greasy, G. S., McKeith, I. G., Kaiser, M., et al. (2015). Resting state in Parkinson's disease dementia and dementia with Lewy bodies: commonalities and differences. Int. J. Geriatr. Psychiatry 30, 1135-1146. doi: 10.1002/gps.4342

Pouratian, N., and Toga, A. W. (2002). "Optical imaging based on intrinsic signals," in Brain Mapping: The Methods, 2nd Edn (San Diego: Academic Press), 97-140. Available online at: http://www.sciencedirect.com/science/article/pii/ B9780126930191500071 [Accessed October 26, 2016].

Prahl, S. (1999). Optical Absorption of Hemoglobin. Available online at: http://omlc.ogi.edu/spectra/hemoglobin/

Raichle, M. E. (2009). A brief history of human brain mapping. Trends Neurosci.32, 118-126. doi: 10.1016/j.tins.2008.11.001

Rosazza, C., and Minati, L. (2011). Resting-state brain networks: literature review and clinical applications. Neurol. Sci. 32, 773-785. doi: 10.1007/s10072-011-0636-y

Saad, Z. S., Gotts, S. J., Murphy, K., Chen, G., Jo, H. J., Martin, A., et al. (2012). Trouble at rest: how correlation patterns and group differences become distorted after global signal regression. Brain Connect. 2, 25-32. doi: 10.1089/brain.2012.0080

Schindelin, J., Arganda-Carreras, I., Frise, E., Kaynig, V., Longair, M., Pietzsch, T., et al. (2012). Fiji: an open-source platform for biological-image analysis. Nat. Methods 9, 676-682. doi: 10.1038/nmeth.2019

Sheffield, J. M., and Barch, D. M. (2016). Cognition and resting-state functional connectivity in schizophrenia. Neurosci. Biobehav. Rev. 61, 108-120. doi: 10.1016/j.neubiorev.2015.12.007

Sizonenko, S. V., Sirimanne, E., Mayall, Y., Gluckman, P. D., Inder, T., and Williams, C. (2003). Selective cortical alteration after hypoxicischemic injury in the very immature rat brain. Pediatr. Res. 54, 263-269. doi: 10.1203/01.PDR.0000072517.01207.87

Smith, S. M., Fox, P. T., Miller, K. L., Glahn, D. C., Fox, P. M., Mackay, C. E., et al. (2009). Correspondence of the brain's functional architecture during activation and rest. Proc. Natl. Acad. Sci. U.S.A. 106, 13040-13045. doi: $10.1073 /$ pnas.0905267106

Smyser, C. D., Dosenbach, N. U. F., Smyser, T. A., Snyder, A. Z., Rogers, C. E., Inder, T. E., et al. (2016a). Prediction of brain maturity in infants using machine-learning algorithms. Neuroimage 136, 1-9. doi: 10.1016/j.neuroimage.2016.05.029

Smyser, C. D., Inder, T. E., Shimony, J. S., Hill, J. E., Degnan, A. J., Snyder, A. Z., et al. (2010). Longitudinal analysis of neural network development in preterm infants. Cereb. Cortex 20, 2852-2862. doi: 10.1093/cercor/bhq035

Smyser, C. D., Snyder, A. Z., Shimony, J. S., Blazey, T. M., Inder, T. E., and Neil, J. J. (2013). Effects of white matter injury on resting state fMRI measures in prematurely born infants. PLOS ONE 8:e68098. doi: 10.1371/journal.pone.0068098

Smyser, C. D., Snyder, A. Z., Shimony, J. S., Mitra, A., Inder, T. E., and Neil, J. J. (2016b). Resting-state network complexity and magnitude are reduced in prematurely born infants. Cereb. Cortex 26, 322-333. doi: 10.1093/cercor/bhu251

Suzuki, K., Yan, P., Wang, F., and Shen, D. (2012). Machine learning in medical imaging. Int. J. Biomed. Imaging 2012:123727. doi: 10.1155/2012/123727
Thorvaldsen, S., Flå, T., and Willassen, N. P. (2010). DeltaProt: a software toolbox for comparative genomics. BMC Bioinformatics 11:573. doi: 10.1186/1471-2105-11-573

Tracy, J. I., and Doucet, G. E. (2015). Resting-state functional connectivity in epilepsy: growing relevance for clinical decision making. Curr. Opin. Neurol. 28, 158-165. doi: 10.1097/WCO.0000000000000178

Ts'o, D. Y., Frostig, R. D., Lieke, E. E., and Grinvald, A. (1990). Functional organization of primate visual cortex revealed by high resolution optical imaging. Science 249, 417-420. doi: 10.1126/science.2165630

van den Heuvel, M. P., Kersbergen, K. J., de Reus, M. A., Keunen, K., Kahn, R. S., Groenendaal, F., et al. (2015). The neonatal connectome during preterm brain development. Cereb. Cortex 25, 3000-3013. doi: 10.1093/cercor/bhu095

Vergara, V. M., Mayer, A. R., Damaraju, E., Kiehl, K. A., and Calhoun, V. (2017). Detection of mild traumatic brain injury by machine learning classification using resting state functional network connectivity and fractional anisotropy. J. Neurotrauma 34, 1045-1053. doi: 10.1089/neu.2016.4526

Wang, K., van Meer, M. P. A., van der Marel, K., van der Toorn, A., Xu, L., Liu, Y., et al. (2011). Temporal scaling properties and spatial synchronization of spontaneous blood oxygenation level-dependent (BOLD) signal fluctuations in rat sensorimotor network at different levels of isoflurane anesthesia. $N M R$ Biomed. 24, 61-67. doi: 10.1002/nbm.1556

Wang, K.-C., Fan, L.-W., Kaizaki, A., Pang, Y., Cai, Z., and Tien, L.-T. (2013). Neonatal lipopolysaccharide exposure induces long-lasting learning impairment, less anxiety-like response and hippocampal injury in adult rats. Neuroscience 234, 146-157. doi: 10.1016/j.neuroscience.2012.12.049

Wang, L., Lai, H. M., Thompson, A. J., and Miller, D. H. (1997). Survey of the distribution of lesion size in multiple sclerosis: implication for the measurement of total lesion load. J. Neurol. Neurosurg. Psychiatr. 63, 452-455. doi: 10.1136/jnnp.63.4.452

Wang, Z., Lu, G., Zhang, Z., Zhong, Y., Jiao, Q., Zhang, Z., et al. (2011). Altered resting state networks in epileptic patients with generalized tonic-clonic seizures. Brain Res. 1374, 134-141. doi: 10.1016/j.brainres.2010.12.034

Warren, J. E., Crinion, J. T., Lambon Ralph, M. A., and Wise, R. J. S. (2009). Anterior temporal lobe connectivity correlates with functional outcome after aphasic stroke. Brain 132, 3428-3442. doi: 10.1093/brain/awp270

White, T. P., Symington, I., Castellanos, N. P., Brittain, P. J., Froudist Walsh, S., Nam, K.-W., et al. (2014). Dysconnectivity of neurocognitive networks at rest in very-preterm born adults. Neuroimage Clin. 4, 352-365. doi: 10.1016/j.nicl.2014.01.005

Wilson, D. A., Hoptman, M. J., Gerum, S. V., and Guilfoyle, D. N. (2011). Statedependent functional connectivity of rat olfactory system assessed by fMRI. Neurosci. Lett. 497, 69-73. doi: 10.1016/j.neulet.2011.04.031

Ye, A. X., AuCoin-Power, M., Taylor, M. J., and Doesburg, S. M. (2016). Disconnected neuromagnetic networks in children born very preterm: disconnected MEG networks in preterm children. Neuroimage Clin. 11, 376-384. doi: 10.1016/j.nicl.2015.08.016

Zernikow, B., Holtmannspoetter, K., Michel, E., Pielemeier, W., Hornschuh, F., Westermann, A., et al. (1998). Artificial neural network for risk assessment in preterm neonates. Arch. Dis. Child Fetal Neonatal. Ed. 79, F129-F134. doi: $10.1136 /$ fn.79.2.F129

Zhan, Y., Paolicelli, R. C., Sforazzini, F., Weinhard, L., Bolasco, G., Pagani, F., et al. (2014). Deficient neuron-microglia signaling results in impaired functional brain connectivity and social behavior. Nat. Neurosci. 17, 400-406. doi: $10.1038 / \mathrm{nn} .3641$

Conflict of Interest Statement: The authors declare that the research was conducted in the absence of any commercial or financial relationships that could be construed as a potential conflict of interest.

The reviewer RL and handling Editor declared their shared affiliation, and the handling Editor states that the process nevertheless met the standards of a fair and objective review.

Copyright (C) 2017 Guevara, Pierre, Tessier, Akakpo, Londono, Lesage and Lodygensky. This is an open-access article distributed under the terms of the Creative Commons Attribution License (CC BY). The use, distribution or reproduction in other forums is permitted, provided the original author(s) or licensor are credited and that the original publication in this journal is cited, in accordance with accepted academic practice. No use, distribution or reproduction is permitted which does not comply with these terms. 